\title{
The Effect of Dose Escalation on the Cost-Effectiveness of Etanercept and Adalimumab with Methotrexate Among Patients with Moderate to Severe Rheumatoid Arthritis
}

\author{
Devin Incerti, PhD; Ervant J. Maksabedian Hernandez, PhD, MPhil; Joseph Tkacz, MS; \\ Jeroen P. Jansen, PhD; David Collier, MD; Mahdi Gharaibeh, PharmD, MSc, PhD; \\ Laura Moore-Schiltz, PhD, MS; and Bradley S. Stolshek, PharmD
}

\begin{abstract}
BACKGROUND: Patients with moderate to severe rheumatoid arthritis (RA) occasionally increase their doses of tumor necrosis factor (TNF) inhibitors, especially the monoclonal antibody origin drugs such as adalimumab and infliximab, after inadequate response to the initial dose. Previous studies have evaluated the cost-effectiveness of various sequences of treatment for RA in the United States but have not considered the effect of dose escalation.
\end{abstract}

OBJECTIVE: To assess the cost-effectiveness of etanercept and adalimumab by incorporating the effect of dose escalation in moderate to severe RA patients.

METHODS: We adapted the open-source Innovation and Value Initiative Rheumatoid Arthritis model, version 1.0 to separately simulate the magnitude and time to dose escalation among RA patients taking adalimumab plus methotrexate or etanercept plus methotrexate from a societal perspective and lifetime horizon. An important assumption in the model was that dose escalation would increase treatment costs through its effect on the number of doses but would have no effect on effectiveness. We estimated the dose escalation parameters using the IBM MarketScan Commercial and Medicare Supplemental Databases. We fit competing parametric survival models to model time to dose escalation and used model diagnostics to compare the fit of the competing models. We measured the magnitude of dose escalation as the percentage increase in the number of doses conditional on dose escalation. Finally, we used the parameterized model to simulate treatment sequences beginning with a TNF inhibitor (adalimumab, etanercept) followed by nonbiologic treatment.

RESULTS: In baseline models without dose escalation, the incremental cost per quality-adjusted life-year of the etanercept treatment sequence relative to the adalimumab treatment sequence was $\$ 85,593$. Incorporating dose escalation increased treatment costs for each sequence, but costs increased more with adalimumab, lowering the incremental costeffectiveness ratio to $\$ 9,001$. At willingness-to-pay levels of $\$ 100,000$, the etanercept sequence was more cost-effective compared with the adalimumab sequence, with probability 0.55 and 0.85 in models with and without dose escalation, respectively.

CONCLUSIONS: Dose escalation has important effects on costeffectiveness and should be considered when comparing biologic medications for the treatment of RA.

J Manag Care Spec Pharm. 2020;26(10):1236-42

Copyright @ 2020, Academy of Managed Care Pharmacy. All rights reserved.

\section{What is already known about this subject}

There are many decision models evaluating the cost-effectiveness of biologic disease-modifying antirheumatic drugs (bDMARDs) for the treatment of moderate to severe rheumatoid arthritis (RA). However, no previous study has evaluated the effect of dose escalation on the cost-effectiveness of these medications.

\section{What this study adds}

This study used empirical estimates of dose escalation for the 2 most commonly prescribed bDMARDs for RA, adalimumab and etanercept, in a simulation model to assess the effect of dose escalation on the cost-effectiveness over a lifetime of etanercept plus methotrexate and adalimumab plus methotrexate from a societal perspective.

Compared with a baseline model without dose escalation, where the etanercept sequence had an incremental cost-effectiveness ratio (ICER) of $\$ 85,593$, a model that incorporated dose escalation showed a decrease in ICER to $\$ 9,001$

Dose escalation can have significant effects on estimates of cost-effectiveness and should be considered when comparing bDMARDs for RA treatment.
$\mathrm{R}$ heumatoid arthritis (RA) is a chronic autoimmune disease that causes pain, swelling, stiffness, and progressive joint damage and affects approximately $0.5 \%-1 \%$ of the population in developed countries. ${ }^{1}$ The disease can have substantial negative effects on health care costs, productivity, caregiver burden, and patient quality of life., ${ }^{2,3}$

The American College of Rheumatology (ACR) recommends that RA patients begin treatment with conventional disease-modifying antirheumatic drugs (cDMARDs), typically methotrexate. ${ }^{4}$ Patients with inadequate response to cDMARDs and more severe RA begin treatment with biologic DMARDs (bDMARDs), most commonly tumor necrosis factor (TNF) inhibitors, with adalimumab and etanercept being the most widely prescribed. ${ }^{5}$ Labels for several bDMARDs allow for dose escalation after inadequate response, defined as either an increase in the dose bolus above a specific threshold or a decreasing interval between boluses. ${ }^{6-8}$ Dose escalation with bDMARDs is not an uncommon 


\section{The Effect of Dose Escalation on the Cost-Effectiveness of Etanercept and Adalimumab with Methotrexate Among Patients with Moderate to Severe Rheumatoid Arthritis}

phenomenon. A systematic review of 33 studies from 2003-2013 reported that inadequate response caused dose escalation in $41.7 \%, 14.9 \%$, and $4.9 \%$ of patients treated with infliximab, adalimumab, and etanercept, respectively. Importantly, the same study showed that dose escalation increased drug costs, RA-related costs, and total costs. ${ }^{9}$

While previous studies have evaluated the cost-effectiveness of various sequences of treatment for RA in the United States, they have not considered the effect of dose escalation. ${ }^{10-14}$ The Second Panel on Cost-Effectiveness in Health and Medicine recommends the use of a societal perspective for the reference case. While payer perspectives are important, we continue to believe that a societal perspective is more relevant, since it considers all costs and outcomes from an intervention, regardless of who pays or experiences it. ${ }^{15}$

In this study, we adapted an open-source decision model for RA, the Innovation and Value Initiative-Rheumatoid Arthritis (IVI-RA) model, so that drug costs were a function of dose escalation. We then used the adapted model to evaluate the effect of dose escalation on the cost-effectiveness of etanercept plus methotrexate relative to adalimumab plus methotrexate..$^{16}$

\section{Methods}

\section{Dose Escalation Data for Etanercept and Adalimumab}

We estimated dose escalation parameters using the IBM MarketScan Commercial and Medicare Supplemental Databases (January 2010-March 2016). The index date was defined as the first claim for adalimumab or etanercept with or without methotrexate between January 2011 and March 2016.

Criteria for inclusion in the statistical analysis were as follows: (a) continuous enrollment for at least 12 months before the index date and at least 12 months after the index date without a previous claim for the index drug in the pre-index period; (b) aged 19 years and older at the index date (i.e., aged 18 years and older in the pre-index period); and (c) an RA diagnosis (International Classification of Diseases, Ninth Revision, Clinical Modification diagnosis code 714.0 and International Classification of Diseases, Tenth Revision codes M05.x or M06.x) any time before or within 30 days after the index date.

Exclusion criteria were (a) a claim for 2 or more different biologics on the index date; (b) a non-RA indication for index therapy any time before or within 30 days after the index date (meaning a claim with a code other than RA); and (c) a malignant neoplasm. Demographic characteristics for patients in our sample are summarized in Table 1.

We defined time to dose escalation as time until the ratio of quantity to days supply was higher than in the index claim. We fitted several parametric survival models by treatment (exponential, Weibull, gamma, Gompertz, loglogistic, lognormal, and generalized gamma). By assessing the model fit using the Akaike and Bayesian information criteria (Supplementary Table 1, available in online article) and

\section{TABLE 1 Demographic Characteristics at Index}

\begin{tabular}{|c|c|c|}
\hline & $\begin{array}{l}\text { Adalimumab } \\
(\mathrm{n}=6,390)\end{array}$ & $\begin{array}{l}\text { Etanercept } \\
(\mathrm{n}=6,586)\end{array}$ \\
\hline Mean age (SD), years & $51.4 \quad(11.2)$ & $51.2 \quad(11.6)$ \\
\hline Female, $\mathrm{n}(\%)$ & $5,002 \quad(78.3)$ & $5,160 \quad(78.3)$ \\
\hline \multicolumn{3}{|l|}{ Insurance plan type, n (\%) } \\
\hline Comprehensive & $442 \quad(6.9)$ & 484 \\
\hline Preferred provider organization & $3,865 \quad(60.5)$ & $3,958 \quad(60.1)$ \\
\hline Health maintenance organization & $586 \quad(9.2)$ & $553 \quad(8.1)$ \\
\hline POS/POS with capitation & $622 \quad(9.7)$ & $752 \quad(11.4)$ \\
\hline Other/unknown & $875 \quad(13.7)$ & $859 \quad(13.0)$ \\
\hline \multicolumn{3}{|l|}{ Payer, n (\%) } \\
\hline Commercial & $5,804 \quad(90.8)$ & $5,993 \quad(91.0)$ \\
\hline Medicare & $586 \quad(9.2)$ & $593 \quad(9.0)$ \\
\hline
\end{tabular}

comparing the parametric to Kaplan-Meier survival curves (Supplementary Figure 1, available in online article), we found that the generalized gamma distribution had the best fit, which was then used in our primary analyses.

We measured the magnitude of dose escalation using the mean percentage increase in the ratio of quantity to days supply of the claim in which dose escalation occurred relative to the initial ratio from the index claim. The quantity can be interpreted as the mean increase in the number of doses during a given period, such as a 6-month model cycle.

\section{Model Overview}

From a societal perspective, we calculated the lifetime costeffectiveness of etanercept and adalimumab using the opensource IVI-RA model, which is a discrete-time individual patient simulation (IPS) with 6-month cycles. Detailed documentation is available online (https://innovationvalueinitiative. github.io/IVI-RA/) and a comprehensive description of the model is published elsewhere. ${ }^{16}$ Originally, the IVI-RA model was designed to estimate the value of alternative sequences of treatment for patients with moderate to severe RA who did not respond to cDMARDs and were naive to bDMARDs or janus kinase inhibitors.

In this study, we compared etanercept plus methotrexate to adalimumab plus methotrexate. We assumed that patients switched to nonbiologic therapy after discontinuation of treatment. Nonbiologic therapy encompasses a range of treatments that may be deemed appropriate by clinicians, typically methotrexate and sulfasalazine. We simulated a heterogeneous population using default values from the model, ${ }^{16}$ where the mean age for the simulated population was 55 years, and $79 \%$ were female, with a mean of 3.3 previous DMARDs used, a mean baseline of the Health Assessment Questionnaire (HAQ) Disability Index score of 1.5, and mean weight for males and females were 89 and $75 \mathrm{~kg}$, respectively. In addition, for the 


\section{FIGURE 1 Percentage of Etanercept and Adalimumab RA Patients with at Least 100\% Dose Increase from} Initial Starting Dose: MarketScan Data 2010-2016

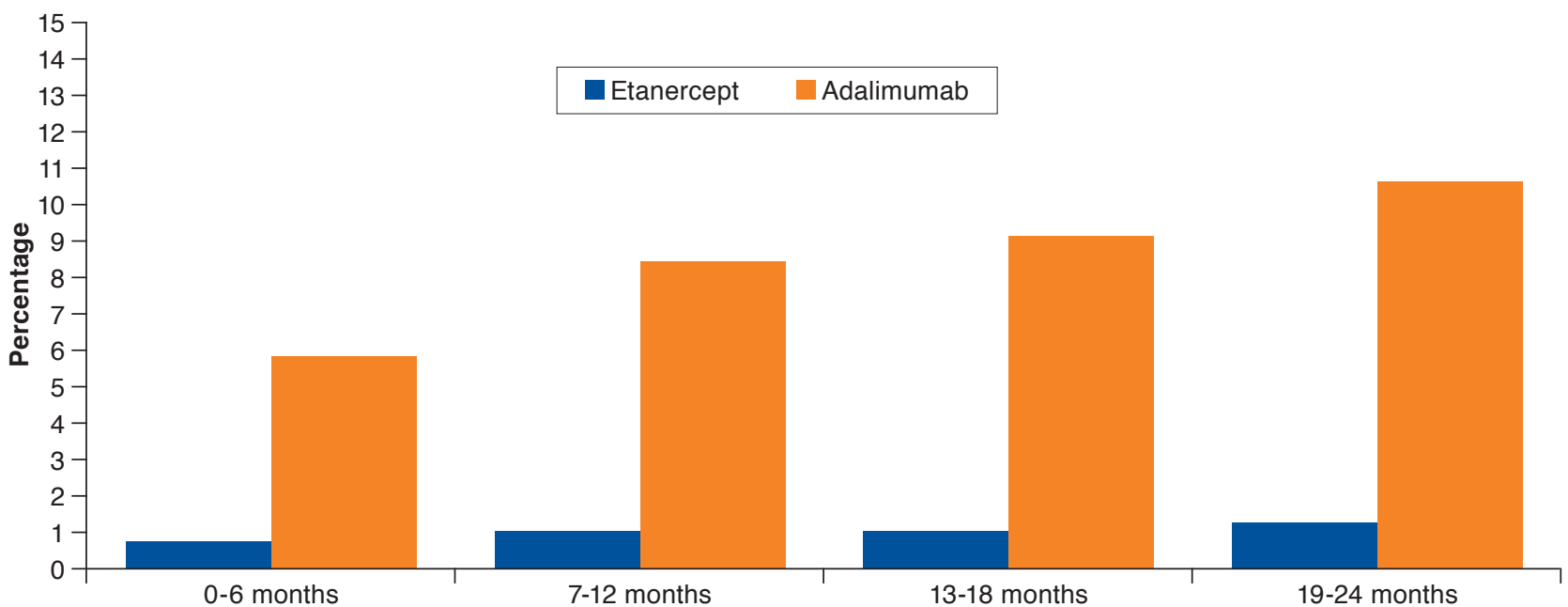

IV-RA model, annual hospitalization days and productivity losses were simulated as a function of HAQ. Health sector costs considered in the models are related to drug acquisition and administration, adverse events, general management of RA, and hospitalization. Drug acquisition and administration costs were calculated separately during the initial treatment phase and the maintenance phase since dosing typically differs. Costs were separated into acquisition costs and infusion costs.

Like most decision models in RA, we measured disease severity using the HAQ score. ${ }^{17-22}$ At the start of the simulation, each patient was assigned a HAQ score. The HAQ trajectory over time was then modeled as a function of the treatment sequence considered. ${ }^{23}$

We used default settings in the model such that (a) the effect of treatment on HAQ during the first 6 months was modeled using the pathway treatment: ACR response to HAQ score; (b) ACR nonresponders discontinued treatment during the first 6 months; (c) a latent class growth model was used to modelterm HAQ progression for patients on nonbiologic therapy; (d) treatment discontinuation from all causes was modeled using a generalized gamma distribution; and (e) a mixture model was used to simulate utility as a function of HAQ. ${ }^{24}$

We quantified parameter uncertainty using probabilistic sensitivity analysis in a 2-stage process: (1) 1,000 parameters were randomly drawn from suitable probability distributions and (2) for each randomly sampled parameter set, 1,000 patients were simulated using the individual patient simulation, and expected values of the quantities of interest were calculated by averaging across patients. Other than the dose escalation adaptation, we used the default parameter values from the IVI-RA model.
All analyses were performed using a societal perspective incorporating health care sector costs and productivity losses. Medication costs, for conventional and biologic treatments in the model, were based on net prices as a function of the wholesale acquisition costs and negotiated discounts in 2019 dollars. Future costs and health outcomes were discounted at a rate of $3 \%$ per year. ${ }^{15}$

\section{Model Adaptation to Incorporate Dose Escalation}

Since the IVI-RA model does not simulate the effect of dose escalation, we created our own version of the model by adapting the underlying code to incorporate this functionality (i.e., "forking" the main IVI-RA model in the GitHub repository). Specifically, we modeled dose escalation in 2 steps. First, we estimated time to dose escalation by randomly drawing a timeto-event from a parametric survival distribution drawn from observational data available from the MarketScan Commercial and Medicare Supplemental Databases (January 2010-March 2016). Second, we measured the mean percentage increase in dose, $k$, conditional on a dose escalation. Following a dose escalation, the number of doses in a 6-month model cycle increased by the estimated percentage increase: dose $e_{\text {new }}=$ dose $_{\text {old }} \times(1+k)$.

\section{Scenario Analysis}

We conducted 2 scenario analyses to test the robustness of the results to the dose escalation parameter estimates. In the first scenario, we considered the effect of the parametric survival models used to extrapolate time to dose escalation beyond the follow-up time available in the observed data. In particular, we ran the simulation for each fitted parametric model. In the second scenario, we assessed the effect of our estimates of the 
FIGURE 2 Effect of Dose Escalation on Cumulative Societal Costs

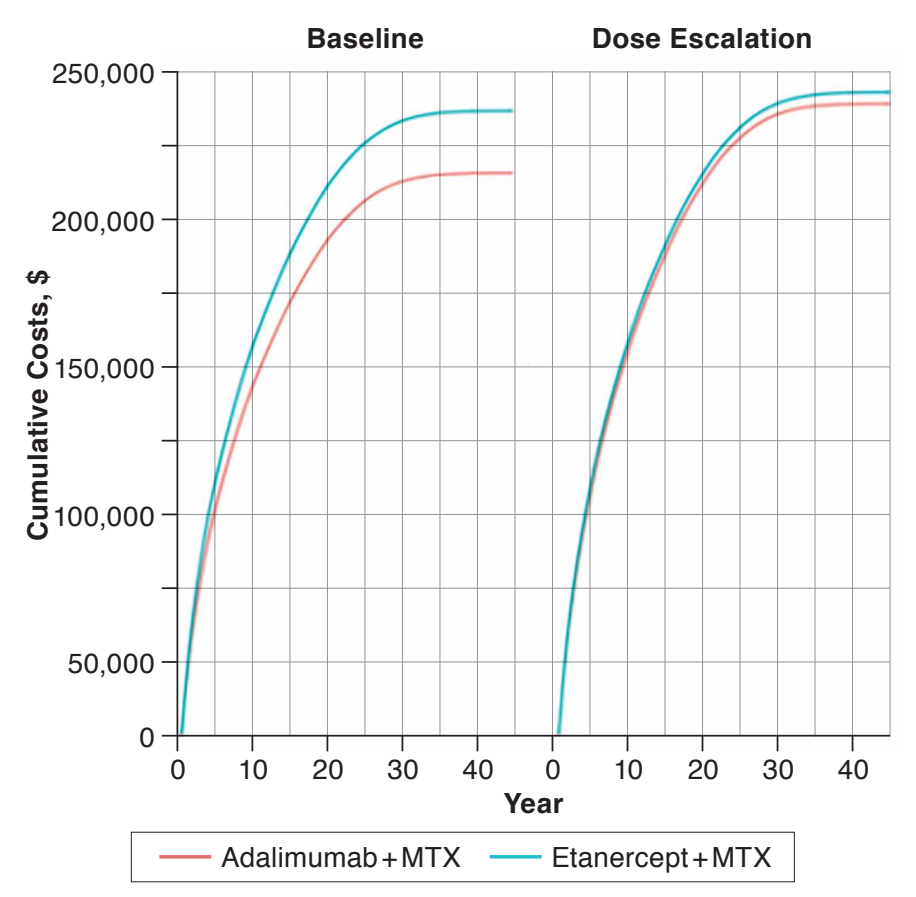

MTX=methotrexate

magnitude of dose escalation by varying values for adalimumab and etanercept at 10\%, 25\%,50\%,75\%, and 100\%. We then ran the model for each of the 25 possible combinations.

\section{Results}

\section{Observational Data for Dose Escalation}

We estimated the Kaplan-Meier estimates of time to any dose escalation for adalimumab and etanercept (not shown). The sample sizes were reasonably large-more than 4,000 patients were at risk at the index date for each bDMARD—which suggests that the survival curves were precisely estimated. The etanercept curve lay above the adalimumab curve, implying that patients escalated doses more quickly with adalimumab. For example, at 3 years, just over $25 \%$ of patients escalated doses with adalimumab, whereas approximately 15\% of patients with etanercept escalated doses by any amount. Differences in dose escalation occurred quickly (within the first year) and remained relatively constant at around 10\% thereafter. For a real-world example of dose escalation, as illustrated in Figure 1, we show the percentage of etanercept and adalimumab RA patients with at least $100 \%$ dose increase from the initial starting dose. Approximately $10 \%$ of all adalimumab patients at risk doubled their dose, while only about $1 \%$ of etanercept patients did so.
For patients who dose escalated, the mean percentage increases in dose escalation over the entire period of observation were $53 \%$ and $24 \%$ for adalimumab and etanercept, respectively. In other words, if a patient was prescribed $40 \mathrm{mg}$ of adalimumab 13 times (i.e., every other week) in a 6-month period before dose escalation, then the $40 \mathrm{mg}$ dose would be prescribed 20 times in the 6-month period after dose escalation. Likewise, a patient prescribed $50 \mathrm{mg}$ of etanercept 26 times (i.e., weekly) in a 6 -month period before dose escalation would be prescribed the same $50 \mathrm{mg}$ dose 32 times after dose escalation.

\section{Cost-Effectiveness Model Analysis}

We ran simulations over a lifetime horizon for a baseline model without dose escalation and a model incorporating dose escalation. In the dose escalation model, $14.7 \%$ and $9.5 \%$ of patients dose escalated with adalimumab and etanercept before treatment discontinuation, respectively.

Figure 2 presents simulated cumulative societal costs. In the baseline model that did not incorporate dose escalation, simulated cumulative societal costs were higher in the etanercept sequence because fewer patients switched treatment than in the adalimumab sequence. Our simulations predicted that the survival curves for time to treatment discontinuation of the etanercept and adalimumab sequences began to separate at 6 months. In this analysis, more patients treated with adalimumab plus methotrexate switched because fewer of these patients achieved an ACR20 response or above. Consequently, etanercept patients remained on therapy longer, suggesting greater effectiveness while remaining on a drug with per dose costs similar to adalimumab. The differences in costs between etanercept and adalimumab began to separate around year 5 and continued separating thereafter.

In the dose escalation scenario that incorporated the estimates of time to dose escalation and the magnitude of dose escalation, cumulative costs in the etanercept sequence relative to the adalimumab sequence decreased considerably because dose escalation was larger with adalimumab. The effect of dose escalation became increasingly important over time, as more patients had a dose escalation. This, in turn, decreased the difference in cumulative costs between the treatment sequences at later time periods.

The incremental cost-effectiveness ratios (ICERs) are reported in Table 2, along mean discounted lifetime quality-adjusted life-years (QALYs) and societal costs. In the baseline model, the etanercept sequence produced slightly more discounted QALYs (6.45 vs. 6.28) but had higher discounted costs $(\$ 303,786$ vs. $\$ 289,587$ ), yielding an ICER of $\$ 85,593$. Simulations with dose escalation produced discounted QALYs that were nearly identical and only differed due to random variation in the IPS. Consistent with Figure 2, costs differences decreased with dose escalation so that discounted costs with the adalimumab 


\section{TABLE 2}

Effect of Dose Escalation on Cost-Effectiveness

\begin{tabular}{l|c|c|c|c}
\hline \multirow{2}{*}{} & \multicolumn{2}{|c|}{ Baseline } & \multicolumn{2}{c}{ With Dose Escalation } \\
\cline { 2 - 5 } & Adalimumab & Etanercept & Adalimumab & Etanercept \\
\hline Discounted costs & $\$ 289,587$ & $\$ 303,786$ & $\$ 307,175$ & $\$ 308,654$ \\
\hline Discounted QALYs & 6.28 & 6.45 & 6.28 & 6.45 \\
\hline ICER & - & $\$ 85,593$ & - & $\$ 9,001$ \\
\hline
\end{tabular}

Note: The baseline scenario does not incorporate dose escalation. The adalimumab treatment sequence is the reference treatment in the ICER calculation. The model was simulated using a lifetime horizon. Outcomes were computed with a $3 \%$ discount rate. Time to dose escalation was modeled using a generalized gamma distribution. Discounted costs included health care sector costs and the costs of lost productivity.

ICER =incremental cost-effectiveness ratio; QALY=quality-adjusted life-year.

$(\$ 307,175)$ and etanercept $(\$ 308,654)$ sequences were similar, decreasing the ICER to $\$ 9,001$.

Figure 3 plots a cost-effectiveness acceptability curve showing the probability that the etanercept sequence was more cost-effective than the adalimumab sequence as a function of the willingness to pay for a QALY. In the baseline scenario, the probability that the etanercept sequence was more costeffective than the adalimumab sequence was at least $50 \%$ if the willingness to pay was greater than $\$ 86,500$, consistent with the ICER; after incorporating dose escalation, this willingness-to-pay threshold decreased to $\$ 10,500$, again consistent with the ICER. At willingness-to-pay levels of $\$ 50,000$ and $\$ 100,000$, the etanercept sequence was more cost-effective in the dose escalation model with probability of 0.70 and 0.85 , respectively.

\section{Scenario Analysis}

The effects of different parametric assumptions for modeling time to dose escalation are reported in Supplementary Table 2 (available in online article). The results suggest that the use of a parametric model is valuable, since in some cases the etanercept sequence was a dominant strategy, meaning that it had lower costs and higher QALYs. This would be expected given that there are considerable differences in the shapes of the parametric survival curves. For instance, the Gompertz and the generalized gamma distributions imply that the proportion of dose escalators will remain relatively flat over time, which decreases the effect of dose escalation. On the other hand, the other distributions imply an upward trend in the proportion of dose escalators, increasing the importance of dose escalation. For these reasons, we made significant efforts to ensure that our choice of parametric survival model was the best fit relative to the other parametric curves in our primary analysis.

We conducted a scenario analyses for the magnitude of dose escalation (figure available upon request). The distribution of incremental costs was generally wide, suggesting there was considerable uncertainty as to whether they were positive or negative. Incremental costs were the largest in absolute value when the differences in the magnitude of dose escalation between the treatment sequences were largest. Simulated incremental costs were the lowest when the magnitude of dose escalation with etanercept was lower than with adalimumab. This scenario analysis showed that cost-effectiveness estimates depended on the difference between treatments in the magnitude of dose escalation and its percentage in absolute terms.

\section{Discussion}

Although previous research has evaluated the cost-effectiveness of bDMARDs in the United States, the effect of dose escalation has not been previously assessed. Our study addressed this question in 2 ways: (1) by empirically estimating the extent of dose escalation for the 2 most commonly prescribed bDMARDs, adalimumab and etanercept, within a large claims database and (2) by using those estimates incorporating dose escalation in a cost-effectiveness simulation model. Dose escalation had an important effect on cost-effectiveness, since the ICER of etanercept relative to a treatment sequence with adalimumab declined from $\$ 85,593$ to $\$ 9,001$. To put our results in perspective, the latest $2020 \mathrm{RA}$ report by the Institute for Clinical and Economic Review found that the ICER of upadacitinib versus adalimumab was $\$ 92,000$ in the base case scenario. ${ }^{25}$

The estimated effects of dose escalation on cost-effectiveness depend on 2 parameters: time to dose escalation and the magnitude of dose escalation among dose escalators. The proportion of patients with any dose escalation within the simulation model with adalimumab (14.7\%) was very similar to the pooled estimate by Moots et al. (2015; 14.9\%), ${ }^{9}$ but the simulated proportion dose escalating with etanercept was higher (9.5\% vs. $4.9 \%)$. This implies that we may have underestimated the cost-effectiveness of the etanercept sequence relative to the adalimumab sequence in a real-world setting (significance between the 2 studies was not tested) because of the higher proportion of patients who dose escalated relative to the pooled estimates, thus increasing our cost estimates for etanercept patients.

Our estimates of the magnitude of dose escalation are consistent with those reported by Moots et al. (2011), who found that among dose escalators, the etanercept dose increased from two to three $25 \mathrm{mg}$ injections per week (50\% increase), and the adalimumab dose increased from a biweekly $40 \mathrm{mg}$ injection to a weekly $40 \mathrm{mg}$ injection (100\% increase). ${ }^{26}$ These estimates are consistent with the ranges considered in our scenario analysis (10\%-100\%) and similar, albeit slightly higher, than our point estimates (53\% for adalimumab, $24 \%$ for etanercept). It is important to note that these percentages reflect only the mean dose escalation among those with any dose escalation, not the mean dose for etanercept and adalimumab RA patients.

Our analysis also highlights the benefits of flexible opensource software. For example, since the IVI-RA model was 


\section{FIGURE 3 Cost-Effectiveness Acceptability Curve}

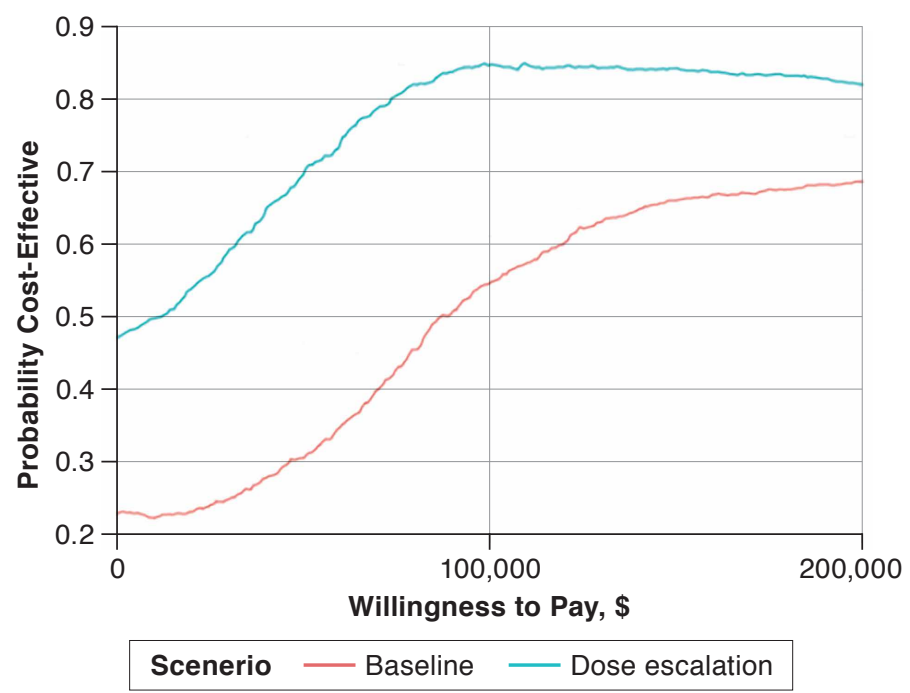

publicly available, we did not need to create a new model from scratch. Instead, we simply added a new feature-the effect of dose escalation-to the existing architecture, which saved time and resources. Open-source models can serve as building blocks for efficiently answering new questions, or even for updating previous analyses. Furthermore, the flexibility of the IVI-RA model allowed us to easily assess the importance of different assumptions in the model, such as the choice of parametric models used to simulate time to dose escalation, as well as assumptions about the magnitude of dose escalation.

\section{Limitations}

This analysis is subject to the limitations of any observational study, but as already discussed, our results are in line with previous research. First, the patient population consisted of individuals with commercial health coverage or private Medicare supplemental coverage; therefore, the results might not be generalizable to RA patients with other types of insurance or without health insurance coverage. Second, patients were identified through administrative claims data rather than through medical records, implying that variables in the analysis, including whether a patient had RA or the exact quantity or days supply for a medication, may have been measured with error. Third, since treatment status was not randomized, differences in dose escalation could have been biased if variables influencing treatment assignment (e.g., baseline health status) also influenced dose escalation.

There are also limitations with the IVI-RA model, which have been described elsewhere. ${ }^{16}$ Here, we will focus on limitations directly related to dose escalation. First, treatment costs in the model are based on net prices as a function of wholesale acquisition costs and negotiated discounts. The model assumes that discounts are measured with uncertainty and range from $20 \%$ to $30 \%$ (mean of $25 \%$ ), but the empirical evidence underlying this range is limited. Nonetheless, the discount rate was slightly lower than the 30\% rate used by the Institute for Clinical and Economic Review in its recent report on the value of treatments for RA. ${ }^{12}$ Second, we did not consider the relationship between dose escalation and effectiveness due to data limitations. The limited evidence around dose and effectiveness suggests that dose escalation happens because of limited effectiveness at the initial dose. ${ }^{27-30}$ Therefore, we believe that our study provides a higher end of the cost-effectiveness of the medications that have dose escalation, in this case, adalimumab. We assume that the initial dose provides full benefits (as reported in the network meta-analysis), which may not be the case when patients experience dose escalation due to lack of response.

\section{Conclusions}

In this study, we assessed the effect of dose escalation on estimates of cost-effectiveness by comparing a treatment sequence of etanercept followed by nonbiologic therapy to a treatment sequence of adalimumab followed by nonbiologic therapy. In our baseline model without dose escalation, the etanercept sequence had an ICER of $\$ 85,593$; conversely, in a model that incorporated dose escalation using parameter estimates from a large commercial claims database, the ICER dropped to $\$ 9,001$. Our study shows that dose escalation can have significant effects on estimates of cost-effectiveness. Payers and decision makers need to make budgetary decisions when looking at different biologic treatments for moderate to severe RA and should consider dose escalation when comparing the cost-effectiveness of bDMARDs for RA.

\section{Authors}

DEVIN INCERTI, PhD, and JEROEN P. JANSEN, PhD, Precision Health Economics, Oakland, California. ERVANT J. MAKSABEDIAN HERNANDEZ, PhD, MPhil; DAVID COLLIER, MD; MAHDI GHARAIBEH, PharmD, MSc, PhD; and BRADLEY S STOLSHEK, PharmD, Amgen, Thousand Oaks, California. JOSEPH TKACZ, MS, and LAURA MOORE-SCHILTZ, PhD, MS, IBM Watson Health, Cambridge, Massachusetts.

AUTHOR CORRESPONDENCE: Ervant J. Maksabedian Hernandez, PhD, MPhil, Amgen, One Amgen Center Dr., Thousand Oaks, CA 91320.Tel.: 805.447.8979; Email:emaksabe@amgen.com. 


\section{The Effect of Dose Escalation on the Cost-Effectiveness of Etanercept and Adalimumab with Methotrexate Among Patients with Moderate to Severe Rheumatoid Arthritis}

\section{DISCLOSURES}

Funding for this study was contributed by Amgen. When this work was conducted, Incerti and Jansen were employees of Precision Health Economics, which received financial support from Amgen. Maksabedian Hernandez, Collier, Gharaibeh, and Stolshek were employees and stockholders of Amgen, and Tkacz and Moore-Schiltz were employees of IBM Watson Health, which received financial support from Amgen.

Some of the results of this work were previously presented as a poster at the 2019 AMCP Managed Care \& Specialty Pharmacy Annual Meeting, March 25-28, 2019, in San Diego, CA

\section{ACKNOWLEDGMENTS}

The authors thank attendants at AMCP Nexus, October 22-25, 2018, in Orlando, FL, for their thoughtful comments. Jessica Ma, PhD (Amgen) and Kripa Madnani (Amgen) provided assistance with writing the manuscript.

\section{REFERENCES}

1. Helmick CG, Felson DT, Lawrence RC, et al. Estimates of the prevalence of arthritis and other rheumatic conditions in the United States. Part I. Arthritis Rheum. 2008;58(1):15-25.

2. Birnbaum H, Pike C, Kaufman R, Marynchenko M, Kidolezi Y, Cifaldi M. Societal cost of rheumatoid arthritis patients in the U.S. Curr Med Res Opin. 2010;26(1):77-90.

3. Lundkvist J, Kastang F, Kobelt G. The burden of rheumatoid arthritis and access to treatment: health burden and costs. Eur J Health Econ. 2008;8(Suppl 2):S49-60.

4. Singh JA, Saag KG, Bridges SL Jr, et al. 2015 American College of Rheumatology guideline for the treatment of rheumatoid arthritis. Arthritis Rheumatol. 2016;68(1):1-26.

5. Atzinger CB, Guo JJ. Biologic disease-modifying antirheumatic drugs in a national, privately insured population: utilization, expenditures, and price trends. Am Health Drug Benefits. 2017;10(1):27-36.

6. Remicade (infliximab). Janssen Biotech. Malvern, PA. Revised November 2013. Available at: https://www.accessdata.fda.gov/drugsatfda_docs/ label/2013/103772s5359lbl.pdf. Accessed August 26, 2010.

7. Humira (adalimumab) injection for subcutaneous use. AbbVie. North Chicago, IL. Revised March 2020. Available at: http://www.rxabbott.com/ pdf/humira.pdf. Accessed August 22, 2019.

8. Actemra (tocilizumab) injection, for intravenous or subcutaneous use. Genentech. South San Francisco, CA. Revised May 2020. Available at: http:// www.gene.com/download/pdf/actemra_prescribing.pdf. Accessed August 22, 2019.

9. Moots RJ, Mays R, Stephens J, Tarallo M. Burden of dose escalation with tumour necrosis factor inhibitors in rheumatoid arthritis: a systematic review of frequency and costs. Clin Exp Rheumatol. 2015;33(5):737-45.

10. Wailoo AJ, Bansback N, Brennan A, Michaud K, Nixon RM, Wolfe F. Biologic drugs for rheumatoid arthritis in the Medicare program: a costeffectiveness analysis. Arthritis Rheum. 2008;58(4):939-46.

11. Carlson JJ, Ogale S, Dejonckheere F, Sullivan SD. Economic evaluation of tocilizumab monotherapy compared to adalimumab monotherapy in the treatment of severe active rheumatoid arthritis. Value Health. 2015;18(2):173-79.

12. Institute for Clinical and Economic Review. Targeted immune modulators for rheumatoid arthritis: effectiveness \& value. Evidence report. April 7, 2017. Available at: https://icer-review.org/wp-content/ uploads/2016/08/NE_CEPAC_RA_Evidence_Report_FINAL_040717.pdf. Accessed August 22, 2020.

13. Jansen JP, Incerti D, Mutebi A, et al. Cost-effectiveness of sequenced treatment of rheumatoid arthritis with targeted immune modulators. J Med Econ. 2017;20(7):703-14.
14. Whittington MD, McQueen RB, Ollendorf DA, et al. Assessing the value of sarilumab monotherapy for adults with moderately to severely active rheumatoid arthritis: a cost-effectiveness analysis. J Manag Care Spec Pharm. 2019;25(1):80-87. Available at: https://www.jmcp.org/doi/10.18553/ jmcp.2019.25.1.080.

15. Neumann PJ, Sanders GD, Russell LB, Siegel JEa, Ganiats TG. Costeffectiveness in Health and Medicine. 2d ed. Oxford University Press; 2016. 16. Incerti D, Curtis JR, Shafrin J, Lakdawalla DN, Jansen JP. A flexible open-source decision model for value assessment of biologic treatment for rheumatoid arthritis. Pharmacoeconomics. 2019;37(6):829-43.

17. Athanasakis K, Tarantilis F, Tsalapati K, Konstantopoulou T, Vritzali E, Kyriopoulos J. Cost-utility analysis of tocilizumab monotherapy in first line versus standard of care for the treatment of rheumatoid arthritis in Greece. Rheumatol Int. 2015;35(9):1489-95.

18. Brennan A, Bansback N, Reynolds A, Conway P. Modelling the costeffectiveness of etanercept in adults with rheumatoid arthritis in the UK. Rheumatology (Oxford). 2004;43(1):62-72.

19. Stephens S, Botteman MF, Cifaldi MA, van Hout BA. Modelling the cost-effectiveness of combination therapy for early, rapidly progressing rheumatoid arthritis by simulating the reversible and irreversible effects of the disease. BMJ Open. 2015;5(6):e006560.

20. Stevenson M, Archer R, Tosh J, et al. Adalimumab, etanercept, infliximab, certolizumab pegol, golimumab, tocilizumab and abatacept for the treatment of rheumatoid arthritis not previously treated with diseasemodifying antirheumatic drugs and after the failure of conventional disease-modifying antirheumatic drugs only: systematic review and economic evaluation. Health Technol Assess. 2016;20(35):1-610.

21. Stevenson MD, Wailoo AJ, Tosh JC, et al. The cost-effectiveness of sequences of biological disease-modifying antirheumatic drug treatment in England for patients with rheumatoid arthritis who can tolerate methotrexate. J Rheumatol. 2017;44(7):973-80.

22. Tosh J, Brennan A, Wailoo A, Bansback N. The Sheffield rheumatoid arthritis health economic model. Rheumatology (Oxford). 2011;50 (Suppl 4):iv26-31.

23. Incerti D, Jansen JP. A description of the IVI-RA Model v2.0. December 16, 2019. Available at: https://innovationvalueinitiative.github.io/IVI-RA/modeldescription/model-description.pdf. Accessed August 26, 2020.

24. Hernández Alava M, Wailoo A, Wolfe F, Michaud K. The relationship between EQ-5D, HAQ and pain in patients with rheumatoid arthritis. Rheumatology (Oxford). 2013;52(5):944-50.

25. Tice J, Kumar V, Chapman R, et al. Janus kinase inhibitors and biosimilars for rheumatoid arthritis. Institute for Clinical and Economic Review, January 9, 2020. Available at: https://icer-review.org/wp-content/ uploads/2019/03/ICER_RA_Final_Evidence_Report_and_Meeting_ Summary_010820.pdf. Accessed August 26, 2020.

26. Moots RJ, Haraoui B, Matucci-Cerinic M, et al. Differences in biologic dose-escalation, non-biologic and steroid intensification among three anti-TNF agents: evidence from clinical practice. Clin Exp Rheumatol. 2011;29(1):26-34

27. Canadian Agency for Drugs and Technologies in Health. Biologics dose escalation for the treatment of rheumatoid arthritis: a review of the clinical and cost-effectiveness. September 22, 2015. Available at: https://www. cadth.ca/sites/default/files/pdf/htis/sep-2015/RC0710\%20Biologics\%20 Dose $\% 20$ Escalation\%20for\%20the\%20Treatment\%20 of\%20Rheumatoid\%20 Arthritis\%20Clinical\%20and\%20Cost-Effectiveness\%20Final.pdf. Accessed August 26, 2020.

28. Ariza-Ariza R, Navarro-Sarabia F, Hernandez-Cruz B, RodriguezArboleya L, Navarro-Compan V, Toyos J. Dose escalation of the anti-TNF-alpha agents in patients with rheumatoid arthritis. A systematic review. Rheumatology (Oxford). 2007;46(3):529-32.

29. Berns M, Hommes DW. Anti-TNF-alpha therapies for the treatment of Crohn's disease: the past, present and future. Expert Opin Investig Drugs. 2016;25(2):129-43.

30. Rahman MU, Strusberg I, Geusens P, et al. Double-blinded infliximab dose escalation in patients with rheumatoid arthritis. Ann Rheum Dis. 2007;66(9):1233-38. 